\title{
Paeonol ameliorates imiquimod-induced psoriasis-like skin lesions in BALB/c mice by inhibiting the maturation and activation of dendritic cells
}

\author{
YUJIAO MENG, MINGXING WANG, XIANGJIANG XIE, TINGTING DI, JINGXIA ZHAO, \\ YAN LIN, XIAOLONG XU, NINGFEI LI, YATING ZHAI, YAN WANG and PING LI \\ Beijing Hospital of Traditional Chinese Medicine, Capital Medical University, Beijing Institute of \\ Traditional Chinese Medicine, Beijing Key Laboratory of Clinic and Basic Research \\ with TCM on Psoriasis, Beijing 100010, P.R. China
}

Received September 5, 2016; Accepted March 9, 2017

DOI: $10.3892 /$ ijmm.2017.2930

\begin{abstract}
Paeonol, an active component derived from the traditional Chinese medicine Cortex Moutan, possesses anti-inflammatory, analgesic, antioxidant and anti-allergic properties. Psoriasis is a chronic, recurrent, inflammatory dermatosis accompanied by excessive activation of Toll-like receptors (TLRs) in dendritic cells (DCs), which are primarily responsible for initiating an immune response. We investigated the effect of paeonol on inflammation in an imiquimod (IMQ)-induced psoriasis-like mouse model and murine bone marrow-derived dendritic cells (BMDCs) stimulated by R848. Mice were intragastrically administered $100 \mathrm{mg} / \mathrm{kg}$ (high), $50 \mathrm{mg} / \mathrm{kg}$ (medium) and $25 \mathrm{mg} / \mathrm{kg}$ (low) paeonol, respectively. We evaluated inflammation of psoriasis-like lesions based on histological changes, protein levels of myeloid differentiation factor 88 (MyD88) and TLR8 in skin lesions by western blotting, and levels of CD11c ${ }^{+}$DCs in skin by immunoassay and in spleens by flow cytometry. Inflammatory cytokines [interleukin (IL)-23, IL-12 and IL-1 $\beta$ ] in skin lesions and BMDCs were also assessed by RT-PCR and ELISA. Application of paeonol decreased IMQ-induced keratinocyte proliferation, and infiltration of $\mathrm{CD}^{+}$cells, while the treatment ameliorated $\mathrm{CD}_{11 \mathrm{c}^{+}}$cells in the spleen and skin, and reduced MyD88 and TLR8 proteins in skin lesions. Paeonol inhibited IMQ-induced mRNA expression of IL-23, but not IL-12 and
\end{abstract}

Correspondence to: Dr Yan Wang or Professor Ping Li, Beijing Hospital of Traditional Chinese Medicine, Capital Medical University, Beijing Institute of Traditional Chinese Medicine, Beijing Key Laboratory of Clinic and Basic Research with TCM on Psoriasis, 23 Art Gallery Back Street, Dongcheng, Beijing 100010, P.R. China

E-mail: wangyan30106@126.com

E-mail: pingli033@sina.com

Key words: psoriasis, paeonol, dendritic cells, Toll-like receptor 7/8, inflammation, immune response
IL-1 $\beta$ in BMDCs, along with significantly lower levels of DCs expressing MHCII, CD80 and CD86 in vitro. These results indicate that paeonol suppresses the maturation and activation of DCs by decreasing MyD88 and TLR8 proteins in the TLR7/8 signaling pathway which finally alleviates psoriasis-like skin lesions. The TLR7/8 signaling pathway in DCs provides an important insight into the mechanism of psoriasis, and paeonol may be a potent therapeutic drug for psoriasis.

\section{Introduction}

Psoriasis is considered as a genetic, immunological skin disease, estimated to affect $2-4 \%$ of the population worldwide $(1,2)$. Histological changes of psoriasis are characterized by hyperproliferation and poor differentiation of epidermal keratinocytes, increased skin vascularization and leukocyte infiltration, including $\mathrm{T}$ cells, macrophages, dendritic cells (DCs) and neutrophils (3). As a central pathogenic player that activates $\mathrm{T}$ cells and produces cytokines and chemokines in psoriasis (4), DCs in the dermis may be responsible for inflammatory infiltrates and development of psoriasis (5).

DCs that serve as surveillance cells in the body, are the most important professional antigen-presenting cells (APCs), and migrate towards immune organs to present processed antigens to $\mathrm{T}$ cells, initiating primary immune responses $(6,7)$. Inflammatory DCs in developing psoriasis are the important source of pro-inflammatory cytokines including interleukin (IL)-23, IL-12, IL-6, IL-1 $\beta$ and tumor necrosis factor- $\alpha$ (TNF- $\alpha)(8,9)$. As known, it is through IL-23 and IL-12 that DCs drive IL-17producing $\mathrm{T}$ cells, such as Th1 and Th22 cells, the activation of which leads to excessive production of psoriatic cytokines such as IL-17 and interferon (IFN) $-\gamma$ mediating effects on keratinocytes to amplify inflammation (10-14). Controlling these pro-inflammatory cytokines in DCs would be a breakthrough for psoriasis treatment.

It is well-known that innate immune cells, such as DCs, recognize invading pathogens by Toll-like receptors (TLRs) and respond appropriately to resolve infections (15). TLRs play important roles in the development of psoriasis, but targeting TLR signaling in DCs remains a challenge for 
treating psoriasis. Imiquimod (IMQ) is a ligand of TLR7/8 and a potent immune activator, which causes activation and maturation of DCs when applied to the skin of mice $(16,17)$. TLR signaling involves the recruitment of adaptor proteins, such as myeloid differentiation factor 88 (MyD88) (18), and controlling the MyD88-dependent signaling pathway should throw new light on the treatment of inflammatory diseases closely related to DCs, such as psoriasis.

Paeonol is a small-molecule compound derived from a Chinese herbal plant Cortex Moutan, widely used as a traditional Chinese medicine (TCM) for alleviation of inflammatory disorders and it has been proved to treat inflammation, allergies and cancer (19). However, its effects on the development and final outcome of these diseases remain unclear. Numerous drugs containing paeonol are applied as prescribed medicines for the treatment of eczema, dermatitis, psoriasis and other skin diseases in China (20). Herein, for the first time, we showed that paeonol alleviated IMQ-induced psoriasis in mice by inhibiting the production of pro-inflammatory cytokines such as IL-23. Bone marrow-derived dendritic cells (BMDCs) stimulated with R848 and treated with paeonol were also investigated. Therefore, control of maturation and activation of DCs by decreasing MyD88 and TLR8 proteins in the TLR7/8 signaling pathway by paeonol could be a novel strategy to treat psoriasis.

\section{Materials and methods}

Psoriatic model in mice. The 8- to 10-week-old-male BALB/c mice (18-20 g) were supplied by Beijing HFK Bioscience Co., Ltd. (Beijing, China) (certification no. SCXK Jing 2014-0004), and maintained with free access to food and water under specific pathogen-free conditions.

The mice were divided into six groups of eight mice each. Five groups were administered a daily topical dose of $42 \mathrm{mg}$ of a cream preparation containing 5\% IMQ (Mingxinlidi Laboratory, China) on their hair-free backs to establish a model of IMQ-induced psoriasis. The control group (Con) received appropriate vaseline. Paeonol (National Institutes for Food and Drug Control, Beijing, China), was dissolved in normal saline (NS) to achieve different concentrations for oral administration. The model group (IMQ) received saline and the methotrexate (MTX) group received $1 \mathrm{mg} / \mathrm{kg}$ MTX, a drug used for psoriasis treatment. The paeonol-high $(\mathrm{PH})$ group received $100 \mathrm{mg} / \mathrm{kg}$ paeonol, the paeonol-medium (PM) group $50 \mathrm{mg} / \mathrm{kg}$ paeonol, and the paeonol-low (PL) group $25 \mathrm{mg} / \mathrm{kg}$ paeonol. All treatments were administered $(0.4 \mathrm{ml} /$ day $)$ from the day IMQ was applied, once a day for a week. After 7 days, the mice were sacrificed by cervical dislocation under sodium pentobarbital anesthesia, while skin lesions and serum samples were collected. All animal experiments were performed in accordance with the Guide for the Care and Use of Laboratory Animals, formulated by the National Institutes of Health (Bethesda, MA, USA), and approved by the Office of the Experimental Animal Management Committee (Beijing, China) and the local animal ethics committee.

Isolation of bone marrow cells and in vitro induction and culture of BMDCs. BMDCs were induced from bone marrow cells of C57/BL6 mice by flushing the femurs and tibiae of
6- to 10-week-old mice with phosphate-buffered saline (PBS). Pooled cells from four limbs were washed in RPMI-1640 medium after lysis of red blood cells, and then plated in 6-well Petri dishes (1x107/well) with RPMI-1640 medium supplemented with $10 \%$ fetal bovine serum (FBS) (Gibco Life Technologies, Grand Island, NY, USA), $1 \%$ penicillin-streptomycin, $20 \mathrm{ng} / \mathrm{ml} \mathrm{IL-4}$ and $20 \mathrm{ng} / \mathrm{ml} \mathrm{GM-CSF}$ (both from PeproTech, Rocky Hill, NJ, USA), cultured for 7 days to induce DCs and the supplemented medium was replaced every day. CD11 ${ }^{+}$DCs were sorted by magnetic beads and seeded in 6-well plates on day 8. BMDCs were then induced to maturation by R848 $(1 \mu \mathrm{g} / \mathrm{ml})$. Different concentrations of paeonol $(75,35.5$ and $17.75 \mu \mathrm{g} / \mathrm{ml})$ were added into the medium, and we collected the cells after $24 \mathrm{~h}$.

Severity scoring of skin inflammation. The severity of skin inflammation was monitored and graded using a modified human scoring system Psoriasis Area Severity Index (PASI). Scaling, thickness and erythema were scored separately on a scale from 0 to 4: 0 , none; 1 , slight; 2 , moderate; 3 , marked; and 4 , very marked. The total score denotes severity of inflammation.

Histopathological examination and immunochemical, immunofluorescence studies. Mice were sacrificed by cervical dislocation under sodium pentobarbital anesthesia after one week. The skin lesions were removed, fixed in $10 \%$ formalin and embedded in paraffin.

Sections $(5-\mu \mathrm{m})$ were stained with hematoxylin and eosin (H\&E). The staining was assessed by light microscope (Olympus, Tokyo, Japan) and epidermal thickness was measured by Image-Pro Plus 6.0 software (Media Cybernetics, Inc., Rockville, MD, USA).

For immunostaining, skin sample sections from the back lesions were stained with anti-rabbit proliferating cell nuclear antigen (PCNA) (Cat. no. ab15497; diluted 1:100), CD3 (Cat. no. ab16669; diluted 1:100) and CD11c (Cat. no. ab33483; diluted 1:500) antibodies (all from Abcam, Cambridge, UK) and staining was assessed using light and fluorescence microscopes (Olympus).

Cell viability assay. The effects of paeonol on cell viability were assessed using the Cell Counting Kit-8 (CCK-8) assay (Dojindo Laboratories, Kumamoto, Japan) according to the manufacturer's instructions. Cells were seeded in 96-well plates and treated with different paeonol concentrations for $24 \mathrm{~h}$. The plates were incubated at $37^{\circ} \mathrm{C}$ in $5 \% \mathrm{CO}_{2}$ for $2 \mathrm{~h}$. The mean optical density (OD) of the cells in each group was used to identify the non-toxic concentration of paeonol.

Real-time polymerase chain reaction (RT-PCR). Total RNA was extracted from skin lesions and BMDCs using TRIzol (InvitrogenLife Technologies,Carlsbad,CA,USA) and purified using a NucleoSpin RNA clean-up kit (Macherey-Nagel, Germany). Following the generation of complementary DNA using an AffinityScript multiple temperature cDNA synthesis kit (Agilent Technologies, Inc., Santa Clara, CA, USA), the relative expression levels of genes were determined with an ABI 7500 Fast Real-Time PCR system using real-time PCR Master Mix (Roche Diagnostics, Indianapolis, IN, USA). The 
Table I. Primers used for RT-PCR.

\begin{tabular}{ll}
\hline \multicolumn{2}{c}{ Primer sequences } \\
\hline IL-23 & F: 5'-ACTCCCCATTCCTACTTCTCCCT-3' \\
& R: 5'-CACTTGCTGCATGAGGAATTGTA-3' \\
IL-1 $\beta$ & F: 5'-TGCCACCTTTTGACAGTGATGA-3' \\
& R: 5'-TGTGCTGCTGCGAGATTTGA-3' \\
IL-12 & F: 5'-TCAACGCAGCACTTCAGAATCACAA-3' \\
& R: 5'-GAAGGCGTGAAGCAGGATGCAGAGC-3' \\
3-actin & F: 5'-CGTTGACATCCGTAAAGACCTC-3' \\
& R: 5'-ACAGAGTACTTGCGCTCAGGAG-3' \\
\hline
\end{tabular}

F, forward; R, reverse; IL, interleukin.

gene-specific primers are listed in Table I. Cycle parameters were as follows: $95^{\circ} \mathrm{C}$ for $10 \mathrm{~min} ; 50$ cycles of $95^{\circ} \mathrm{C}$ for $15 \mathrm{sec}$, and $60^{\circ} \mathrm{C}$ for $60 \mathrm{sec}$. The $\beta$-actin gene was used as a reference to normalize the data that was quantitatively analyzed using the $2^{-\Delta \Delta \mathrm{Cq}}$ method.

Western blotting. Skin samples were lysed and the protein was resolved by $10 \%$ sodium dodecyl sulfate-polyacrylamide gel electrophoresis (SDS-PAGE). The membrane fractions were incubated with MyD88 (4283; Cat. no. 4283; Cell Signaling Technology, Inc., Danvers, MA, USA), rabbit-TLR8 (Cat. no. ab180610; Abcam) and mouse anti- $\beta$-tubulin (Cat. no. YM3030; Immunoway) antibodies, followed by IRDye 700DX- or 800DX-conjugated secondary antibodies (Rockland Immunochemicals Inc., Gilbertsville, PA, USA). Immunofluorescence was assessed by Odyssey infrared imaging system (LI-COR Biosciences, Lincoln, NE, USA).

Flow cytometric analysis. Spleen samples from each group were harvested to determine whether the frequency of DCs was altered in the spleens of the IMQ-induced mouse model following treatment with paeonol. Following, the samples were minced through a $70-\mu \mathrm{m}$ mesh to obtain single-cell suspensions, and $1 \times 10^{6}$ cells were stained with fluorescein isothiocyanate (FITC)-conjugated mouse anti-CD11c. Samples were analyzed using flow cytometry (FCM).

To determine whether paeonol altered the ratio of mature and activated DCs induced by R848 in vitro, BMDCs from different groups were suspended in PBS, and then stained with anti-CD11c-PE (Cat. no. ab155349), anti-MHCII-FITC (Cat. no. ab93561), anti-CD80-FITC (Cat. no. ab24860) and anti-CD86-FITC (Cat. no. ab24862) antibodies at $4^{\circ} \mathrm{C}$ for $30 \mathrm{~min}$ in the dark. Cells were washed once with PBS and analyzed with FCM.

ELISA. BMDCs were seeded in 12-well culture plates at a density of $1 \times 10^{6}$ cells $/ \mathrm{ml}$ and pretreated with various concentrations $(75,35.5$ and $17.75 \mu \mathrm{g} / \mathrm{ml})$ of paeonol for $24 \mathrm{~h}$, followed by R848 (Cat. no. SML0196; Sigma, St. Louis, MO, USA) $(1 \mu \mathrm{g} / \mathrm{ml})$ for $24 \mathrm{~h}$. The levels of IL-12p40 and IL-12p70 in the supernatants were determined using mouse ELISA kits according to the manufacturer's instructions, with stan-

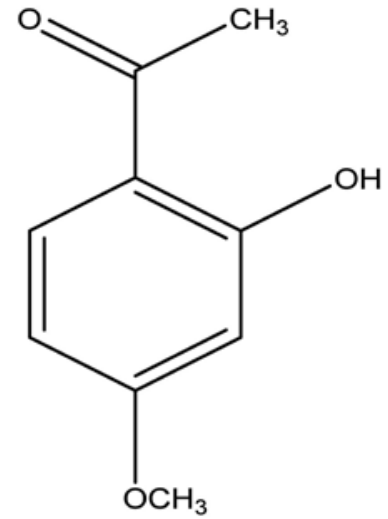

Figure 1. Chemical structure of paeonol.

dard curves made from purified recombinant IL-12p40 and IL-12p70 at various dilutions.

Statistical analysis. Results are expressed as mean \pm SD. Differences between groups were evaluated with Student's t-test (for comparisons between two samples) or by a one-way analysis of variance (ANOVA) (for comparisons of multiple samples) using the SPSS 15.0 software (SPSS, Inc., Chicago, IL, USA). A p-value $<0.05$ was considered to indicate a statistically significant difference.

\section{Results}

Paeonol improves psoriatic lesions in a mouse model by inhibiting proliferation and differentiation of keratinocytes. We initially investigated the effect of paeonol (Fig. 1) on the psoriatic mouse model induced by IMQ through morphological observations and pathological slices. Typical erythema, scaling and thickening were observed in the IMQ-induced skin lesions as compared to the control group, while paeonol significantly inhibited these pathological changes in a dose-dependent manner (Fig. 2A and B).

Skin treated with IMQ demonstrated pathological changes of the epidermal cuticle, including significant parakeratosis, acanthosis and perivascular infiltration of inflammatory cells in the upper dermis, a phenotype typical of human psoriatic skin. Paeonol significantly reduced the thickness of the epidermis, and attenuated IMQ-induced psoriasis (Fig. 2C and E).

PCNA is expressed in proliferative cells, especially basal cells. Expression of PCNA was reduced in the skin lesions of the paeonol-treated mice, suggesting that paeonol reduces IMQ-induced proliferation of keratinocytes (Fig. 2D and F) and effectively ameliorates IMQ-induced keratinocyte differentiation.

Paeonol downregulates inflammatory infiltration especially mature and activated DCs along with pro-inflammatory cytokines in a mouse model of psoriasis. CD3 is mainly expressed in $\mathrm{T}$ lymphocytes and appears as brown particles after immunohistochemistry with the DAB chromogenic agent. Infiltration of $\mathrm{CD}^{+}$cells was observed in IMQ-induced lesions but was reduced in the paeonol-treated mice. Fig. 3B shows the expression of $\mathrm{CD}^{+}$cells, not IL-23 mRNA in the skin lesions, and it is the statistical data for Fig. 3A. 
A

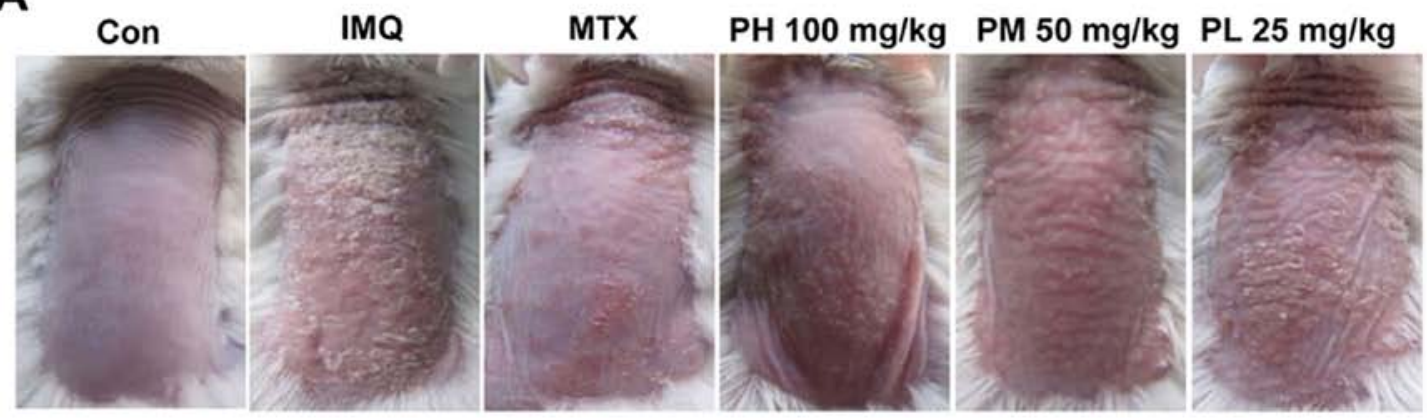

B
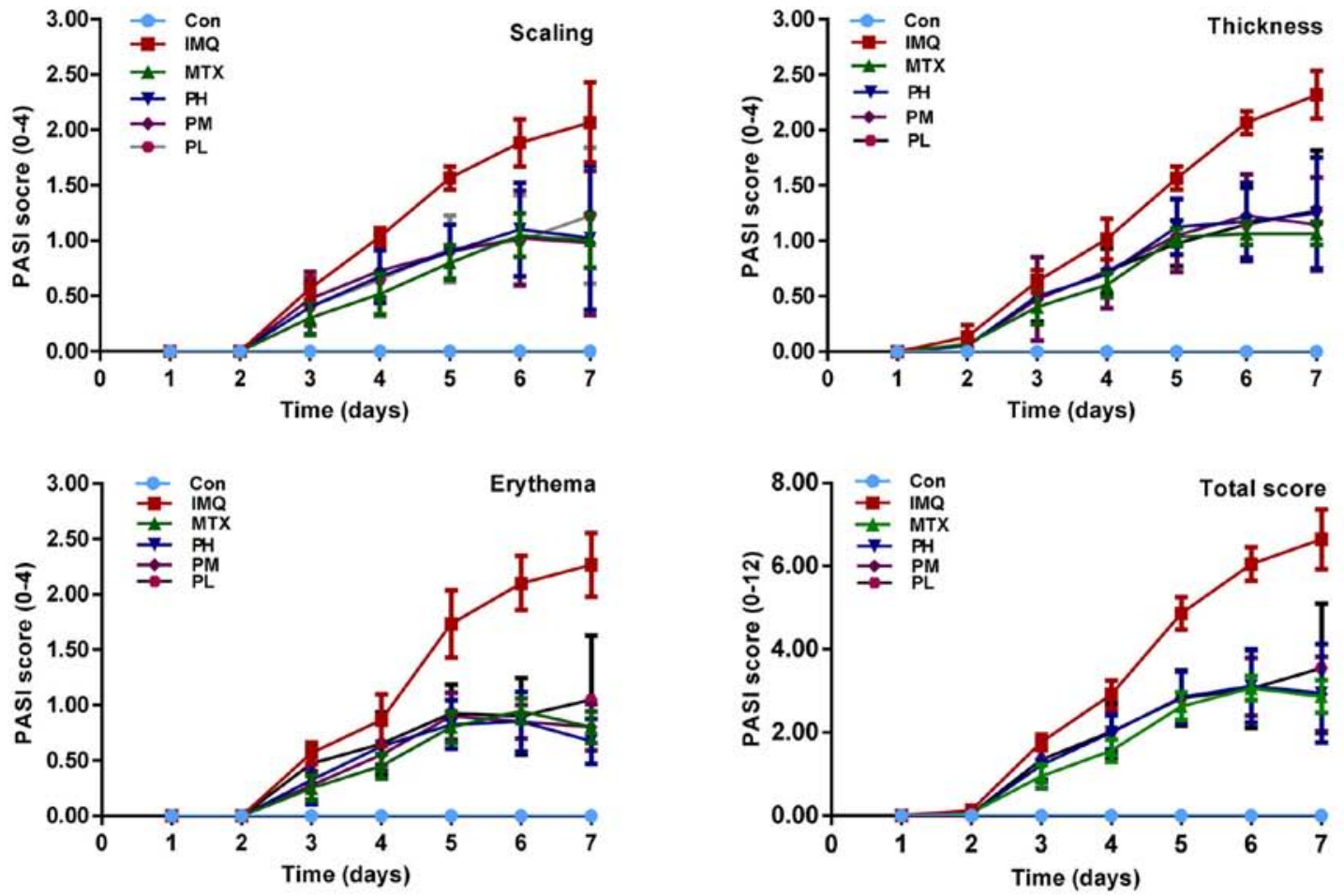

C
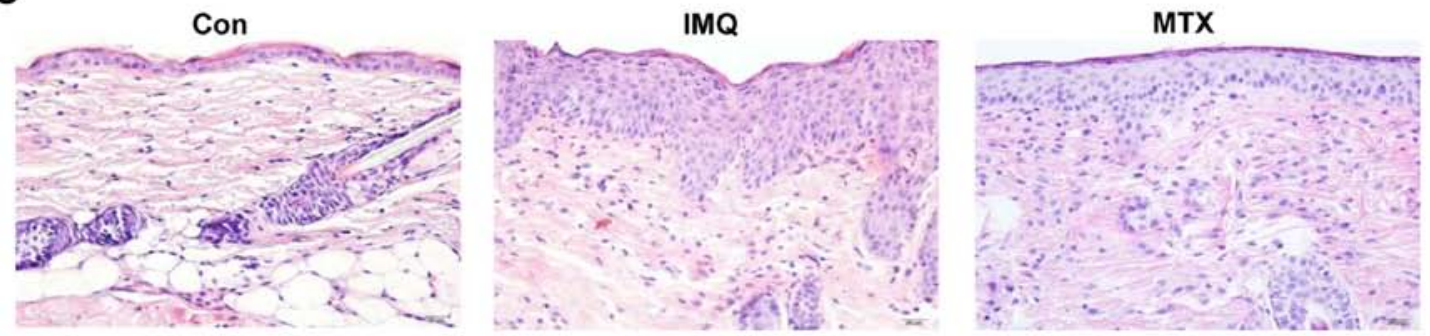

$\mathrm{PH} 100 \mathrm{mg} / \mathrm{kg}$

PM $50 \mathrm{mg} / \mathrm{kg}$

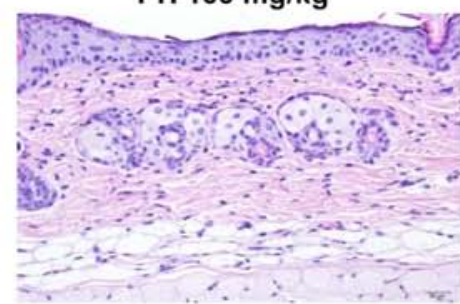

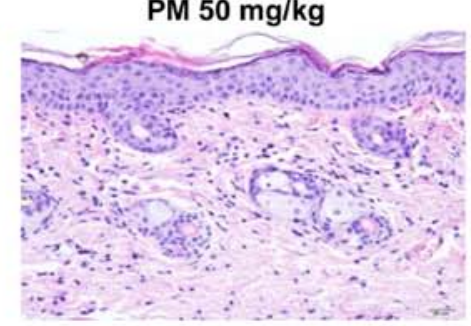

\section{PL $25 \mathrm{mg} / \mathrm{kg}$}

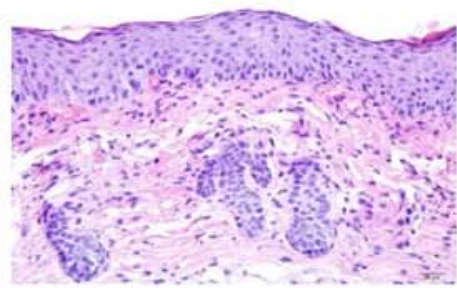

Figure 2. Paeonol exerts improvement on imiquimod (IMQ)-induced psoriasis in mice. Psoriatis-like skin was observed in mice after one week following topical application of IMQ, but not in the control animals (Con). Animals administered 100 (PH), 50 (PM) and $25 \mathrm{mg} / \mathrm{kg} \mathrm{paeonol}(\mathrm{PL}) \mathrm{or} 1 \mathrm{mg} / \mathrm{kg} \mathrm{metho-}$ trexate (MTX) exhibited ameliorated symptoms $(0.4 \mathrm{ml} / \mathrm{day}$ ), while the mouse model fed with the same amount of saline did not. (A) Comparision of the back skin in the different groups after IMQ exposure for a week. (B) Psoriasis Area Severity Index (PASI) score of skin lesions in the different groups including scaling, thickness and erythema on a scale from 0 to 4 and the total score is indicated. (C) Phenotypical presentation and corresponding histological analyses (H\&E staining, $\mathrm{x} 200$ ) of mouse back skin and epidermal thickness, with obvious acanthosis, parakeratosis, pustules and desquamation in the model groups. 
D

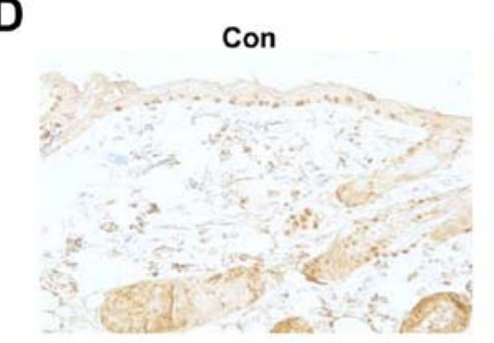

$\mathrm{PH} 100 \mathrm{mg} / \mathrm{kg}$

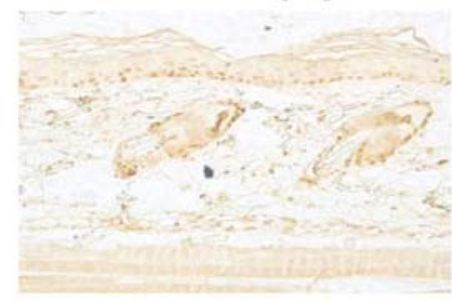

$\mathbf{E}$

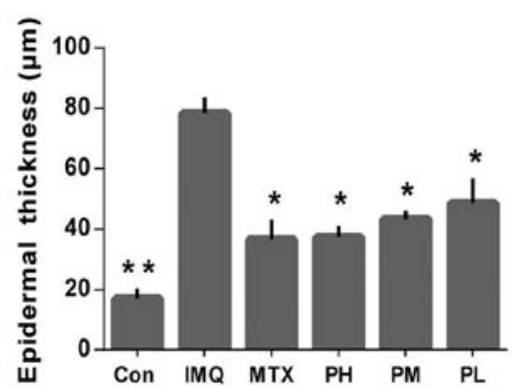

IMQ

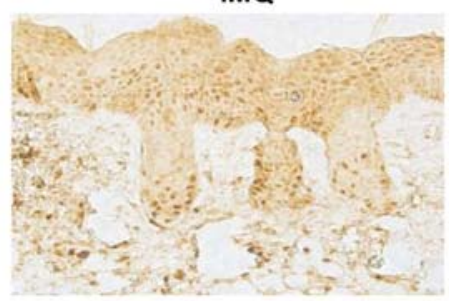

$\mathrm{PM} 50 \mathrm{mg} / \mathrm{kg}$

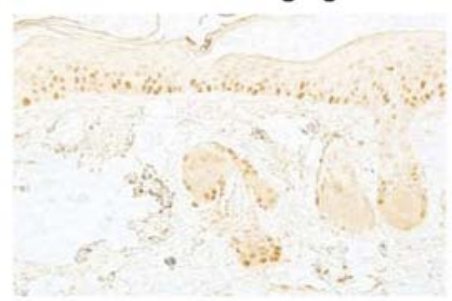

MTX

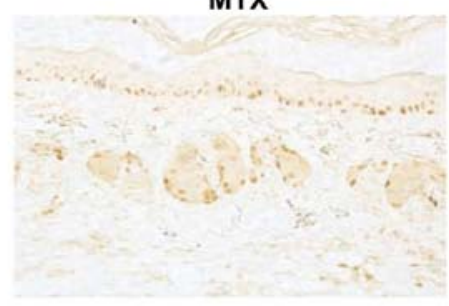

PL $25 \mathrm{mg} / \mathrm{kg}$

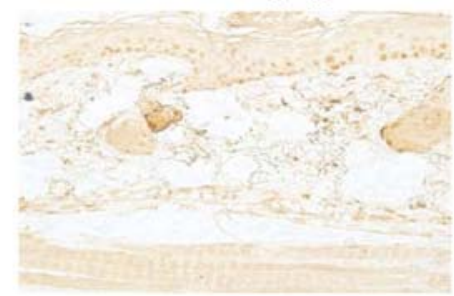

$\mathbf{F}$

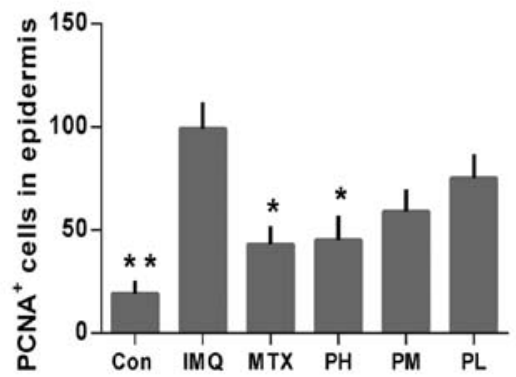

Figure 2. Continued. (D) IHC staining (x200) for proliferating cell nuclear antigen (PCNA) (brown) in mouse back skin. (E) Epidermal thickness of each group. (F) Statistical analysis of the number of $\mathrm{PCNA}^{+}$cells in the epidermis. Data are expressed as the mean $\pm \mathrm{SD}$ ( $\mathrm{n}=6 /$ experiment). ${ }^{*} \mathrm{p}<0.05$ and ${ }^{* * *} \mathrm{p}<0.01$ vs. mouse model.

The numbers of $\mathrm{CD} 11 \mathrm{c}^{+} \mathrm{DCs}$ in the dorsal skin and spleens of IMQ-induced mice were detected by immunofluorescence assay (IFA), which showed a major increase in these cells in the dorsal skin (Fig. 3C and D) as well as in the spleens, which were markedly increased (Fig. 3E and F).

Compared with the control group, the mRNA expression level of IL-23 was markedly increased in the IMQ-induced psoriatic skins (Fig. 3G). As expected, expression in the group treated with paeonol was significantly decreased.

Paeonol decreases MyD88 and TLR8 proteins in a mouse model of psoriasis. We further explored how paeonol inhibits the TLR7/8 signaling pathway, and found lower protein expression of MyD88 and TLR8 in skin lesions of the paeonoltreated group as compared to these levels in the IMQ-induced model (Fig. 4A and B).

Paeonol inhibits the maturation and activation of BMDCs. We stimulated BMDCs with $1 \mu \mathrm{g} / \mathrm{ml}$ R848. Surface markers of DCs including CD11c, MHCII, CD80 and CD86 were detected by flow cytometry. MHCII, CD80 and CD86 were downregulated in the paeonol-treated mice (Fig. 5).

Paeonol decreases the expression of pro-inflammatory cytokines secreted by mature BMDCs. The mRNA levels of IL-23, IL-12 and IL-1 $\beta$ were measured by RT-PCR and related cytokines were assessed by ELISA. RT-PCR showed that IL-23 was significantly suppressed, while IL-12 and IL-1 $\beta$ were enhanced after paeonol treatment in a dosedependent manner, while cytokine levels were decreased by paeonol (Fig. 6A and B).

\section{Discussion}

Psoriasis is a chronic autoimmune disease mediated by immune cells and molecules, with persistent inflammation and hyperplasia (21). Suppression of T cell responsiveness by influencing the phenotype and function of DCs, the most powerful APCs, remains one of the most effective treatment approaches (22). As bridges that link innate and adaptive immunity, activated and mature DCs lead to the next phase of immune reactions under physiological circumstances, removing pathogens from the body (23). However, inappropriate activation and maturation of DCs are closely related to the development of immune disorders and multiple pro-inflammatory cytokines such as IL-23, IL-12 and IL-1 $\beta$ in the psoriatic skin are secreted by DCs (24). Symptoms could be relieved if signaling pathways of DCs are inhibited.

TLRs play a central role in the recognition and response to invading pathogens by immune cells, leading to the production 
A
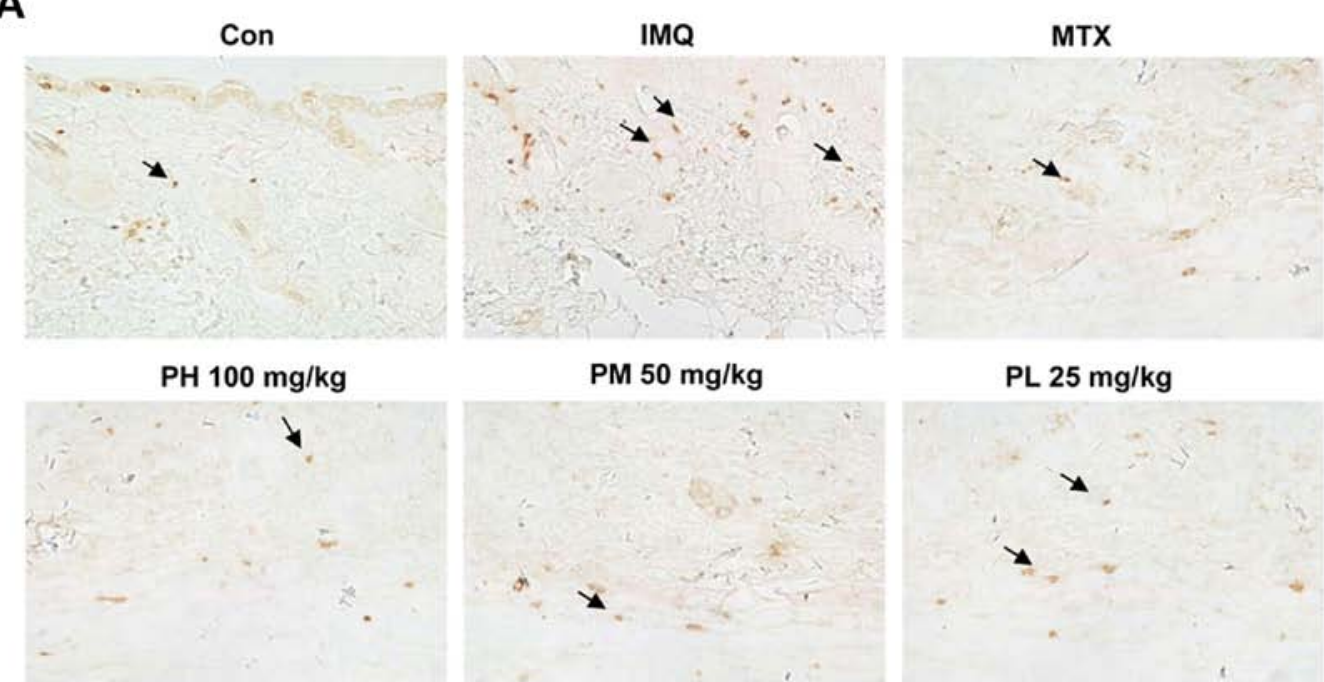

PM $50 \mathrm{mg} / \mathrm{kg}$

PL $25 \mathrm{mg} / \mathrm{kg}$
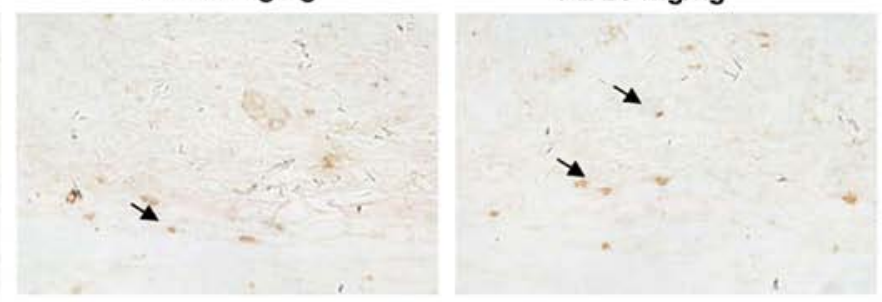

B

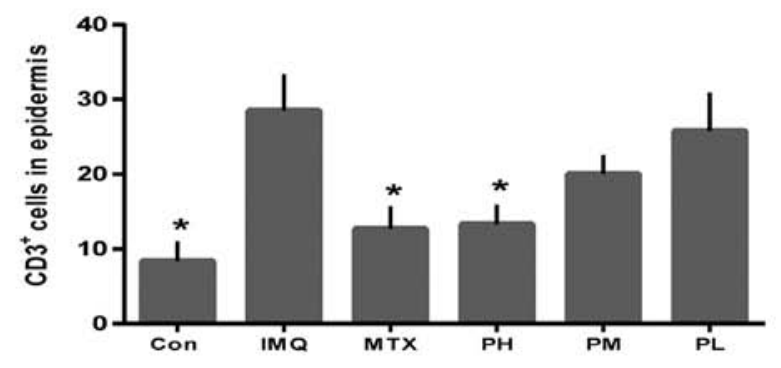

C

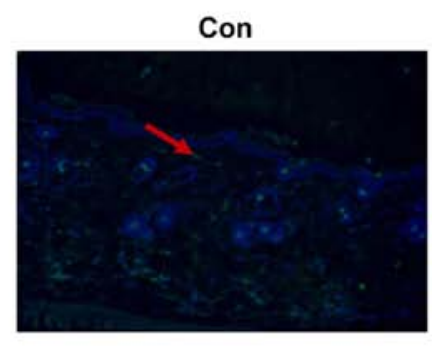

PH $100 \mathrm{mg} / \mathrm{kg}$

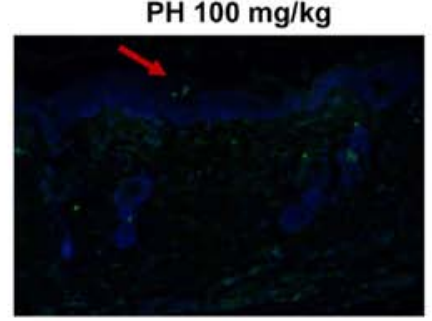

IMQ

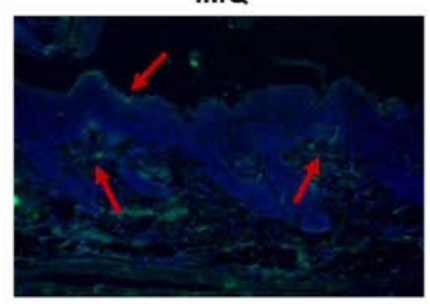

$\mathrm{PM} 50 \mathrm{mg} / \mathrm{kg}$

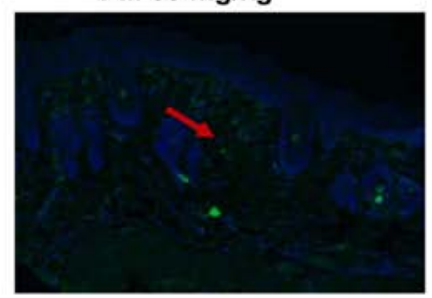

MTX

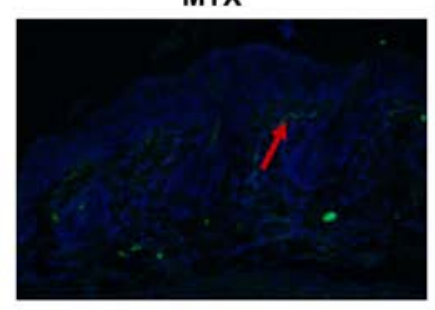

PL $25 \mathrm{mg} / \mathrm{kg}$

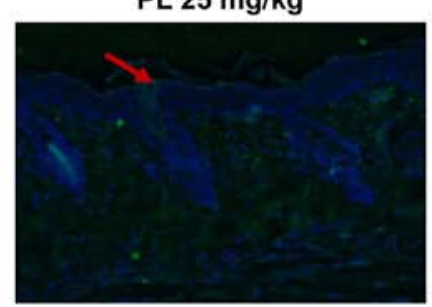

Figure 3. Paeonol alleviates inflammatory cell infiltration in psoriasis-like lesions and spleens of psoriatic models and decreases mRNA expression of psoriasis-related cytokines. (A) $\mathrm{CD}^{+}$cells in imiquimod (IMQ)-induced mice with psoriasis (brown color, $\mathrm{x} 200$ ). (B) Description of $\mathrm{CD} 3^{+}$statistical data. (C) IF staining (x200) of CD11 $\mathrm{c}^{+}$cells (green) in skin. " $\mathrm{p}<0.05$ vs. the mouse model.

of pro-inflammatory cytokines and chemokines (25). The TLR7/8, signaling pathway, which includes recruitment of MyD88, could be an important target for treating psoriasis. The role of MyD88 protein in the TLR7/8 signaling pathway has been established $(26,27)$. Blocking of the MyD88-dependent signaling pathway aids in the treatment of psoriasis.

IMQ is a TLR7/8 agonist, and the IMQ-induced mouse model is a classic model of psoriasis (28). Mice administered paeonol showed lower scores for PASI, thinner epidermis and decreased keratinocyte proliferation and differentiation, which were positively correlated with the paeonol concentration (Fig. 2). We believe that paeonol can be potentially used for treating psoriasis in a dose-dependent manner.

The role of the IL-23/Th17 axis in the development of psoriasis is a current focus of research (29). However, we are more interested in the upstream pathway. Research has shown 
D

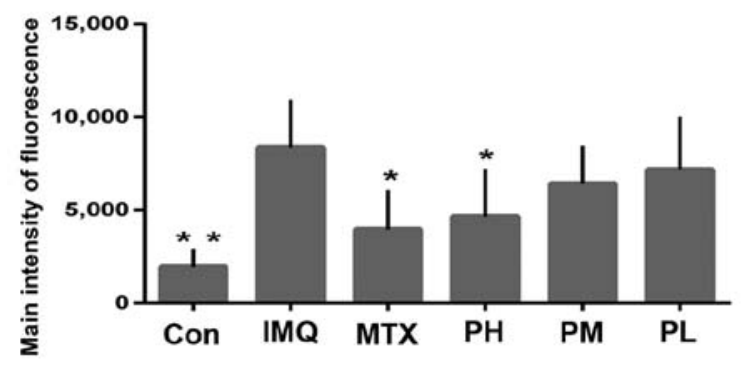

$\mathbf{F}$

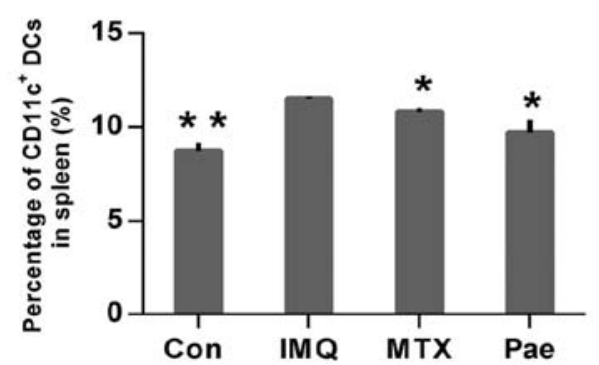

E
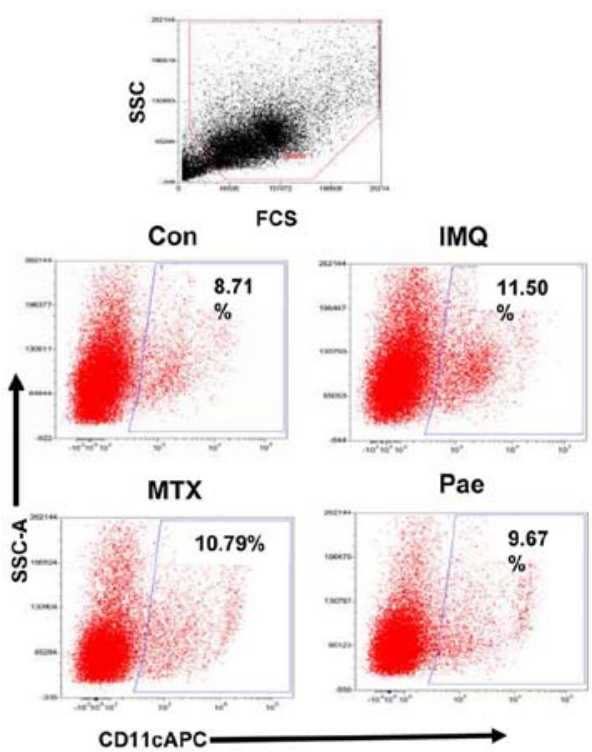

G

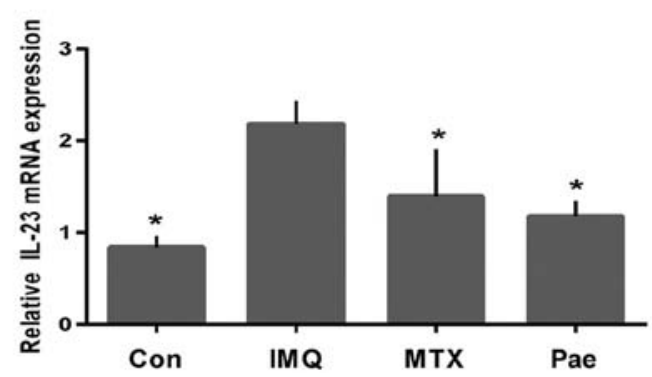

Figure 3. Continued. (D) Statistical analysis of the mean fluorescence intensity in C. (E) Percentage of CD11 ${ }^{+}$cells in the spleens of the IMQ-mouse model by flow cytometry (FCM). (F) Statistical analysis of the percentage of CD11 $\mathrm{c}^{+}$cells in the spleen. (G) RT-PCR of IL-23 in skin lesions from the psoriasis-like mice induced by IMQ. Data are expressed as the mean $\pm \mathrm{SD}$ ( $\mathrm{n}=6 /$ experiment). ${ }^{*} \mathrm{p}<0.05$ and ${ }^{* *} \mathrm{p}<0.01$ vs. the mouse model.
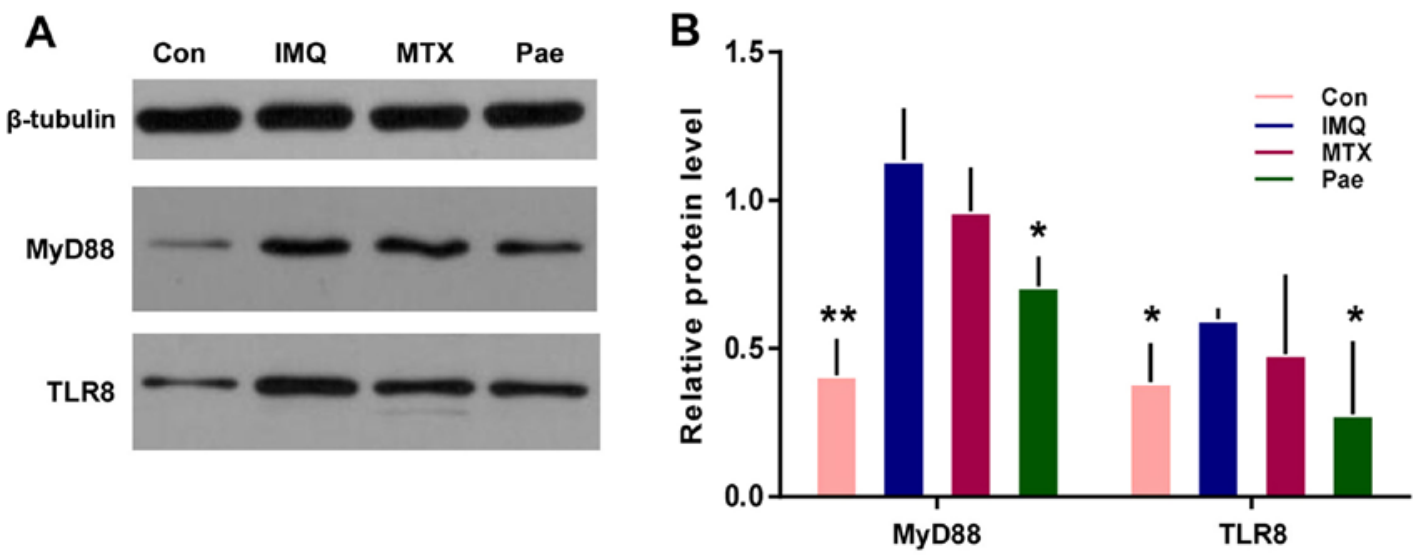

Figure 4. Paeonol decreases myeloid differentiation factor 88 (MyD88) and Toll-like receptor 8 (TLR8) proteins in the skin lesions of the psoriatic mouse model, and inhibits activation of the TLR7/8 signaling pathway. (A) The levels of MyD88 and TLR8 in paeonol-treated mice were assessed by western blotting. Immunoblotting showing the bands detected for various proteins. (B) Quantitation of A after normalization with $\beta$-tubulin. Data are expressed as the mean $\pm \mathrm{SD}\left(\mathrm{n}=6 /\right.$ experiment). ${ }^{*} \mathrm{p}<0.05$ and ${ }^{* *} \mathrm{p}<0.01$ vs. the mouse model.

that $\mathrm{CD} 11 \mathrm{c}$ is regarded as the correct marker of myeloid DCs (30) and the number of CD11 $\mathrm{c}^{+}$cells was lower in the spleens of the paeonol-treated mice (Fig. 3), consistent with the data from the immunofluorescence assay of skin lesions indicating that paeonol decreased the number of DCs. IL-23, mostly secreted by mature and activated DCs, is responsible for the local inflammation of psoriatic skin, leading us to focus on DCs (31). Other studies have shown that pro-inflammatory factors such as TNF- $\alpha$, MCP-1, IL- $1 \beta$ and IL- 6 were dosedependently reduced by paeonol treatment in vivo (32-35). 
A

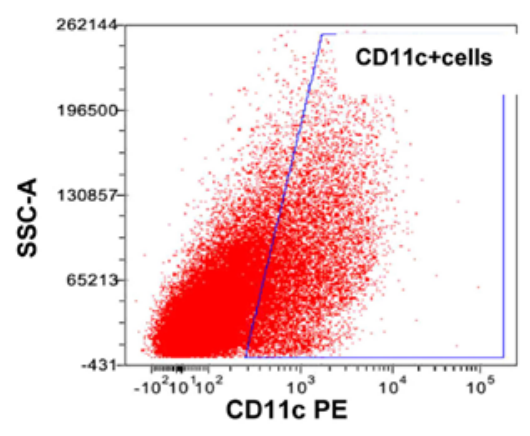

C

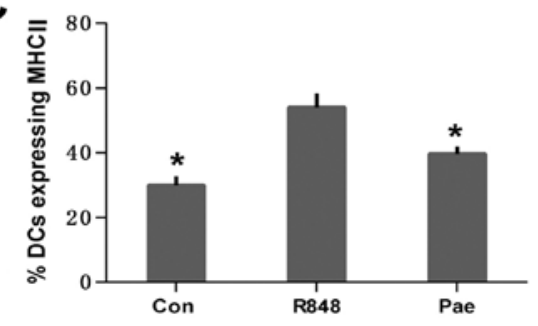

E

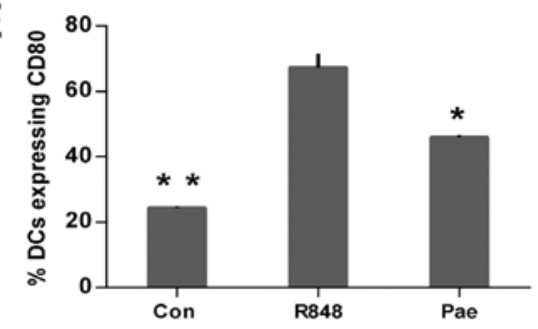

G

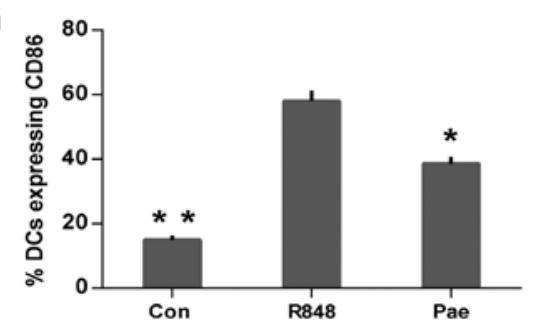

B

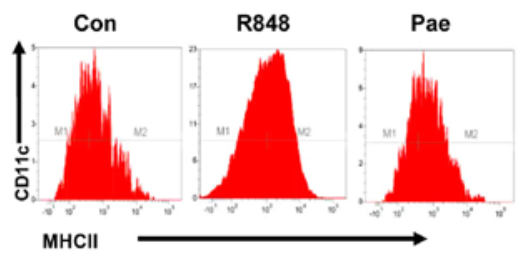

D

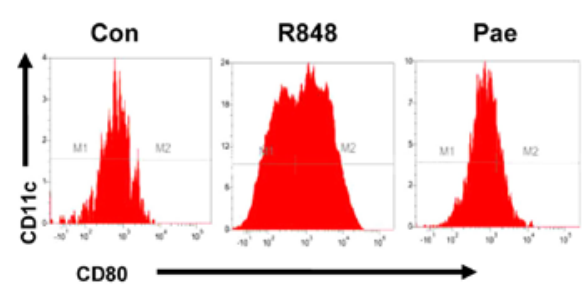

$\mathbf{F}$

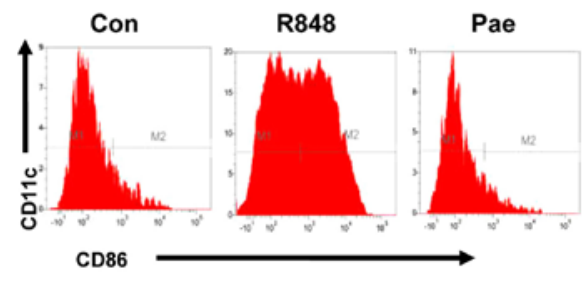

Figure 5. Paeonol inhibits maturation and activation of bone marrow-derived dendritic cells (BMDCs). (A) Representative dot plots showing the percentage of CD11 $\mathrm{c}^{+}$BMDCs (gate on the right). (B and C) Chart and bar graph showing the rates of BMDCs expressing MHCII stimulated by R848. (D and E) Chart and bar graph showing the rates of BMDCs expressing CD80 stimulated by R848. (F and G) Chart and bar graph showing the rates of BMDCs expressing CD86 stimulated by R848. Data are expressed as the mean $\pm \mathrm{SD}\left(\mathrm{n}=6 /\right.$ each group). ${ }^{\mathrm{p}}<0.05$ and ${ }^{* *} \mathrm{p}<0.01$ vs. the mouse model.

Results from skin lesions showed that expression of IL-23 mRNA decreased in a dose-dependent manner (Fig. 3G), as previously reported. We further detected the expression of MyD88 and TLR8 proteins (Fig. 4), which were significantly reduced in the paeonol-treated group.

BMDCs were collected for further study concerning the frequency and the phenotype of DCs. MHCII, CD80 and CD86 are all specific markers for mature DCs (36). BMDCs cultured for 1 week were stimulated with R848, a TLR8 agonist that induces DC maturation for $24 \mathrm{~h}$, and were then treated with paeonol. The number of marker-positive cells following paeonol therapy was significantly reduced as compared to the model group (Fig. 5). Results from the in vitro experiments indicated that paeonol improved the clinical symptoms of psoriasis by suppressing the maturation and activation of DCs. CD80 and
CD86 are induced in an MyD88-dependent manner in the TLR7 signaling pathway, and the IMQ-induced mouse model is characterized by activated TLR7. Moreover, R848 used in our in vitro experiment is a TLR8 agonist. Meanwhile, expression of MyD88 and TLR8 proteins was significantly reduced in the paeonol-treated group. Thus, paeonol plays a role in anti-inflammation of psoriasis by inhibiting maturation and activation of DCs through the TLR7/8 signaling pathway in DCs.

In order to validate the suppressive effects on inflammation in BMDCs, we also detected the expression of IL-23, IL-12 and IL-1 $\beta$, cytokines that cause specific T cell responses (36). Results from the BMDCs showed that expression of IL-23 mRNA decreased in a dose-dependent manner, consistent with our results in the skin lesions (Fig. 6). Meanwhile, ELISA for IL-12p40 in BMDCs indicated that the levels of IL-23 
A
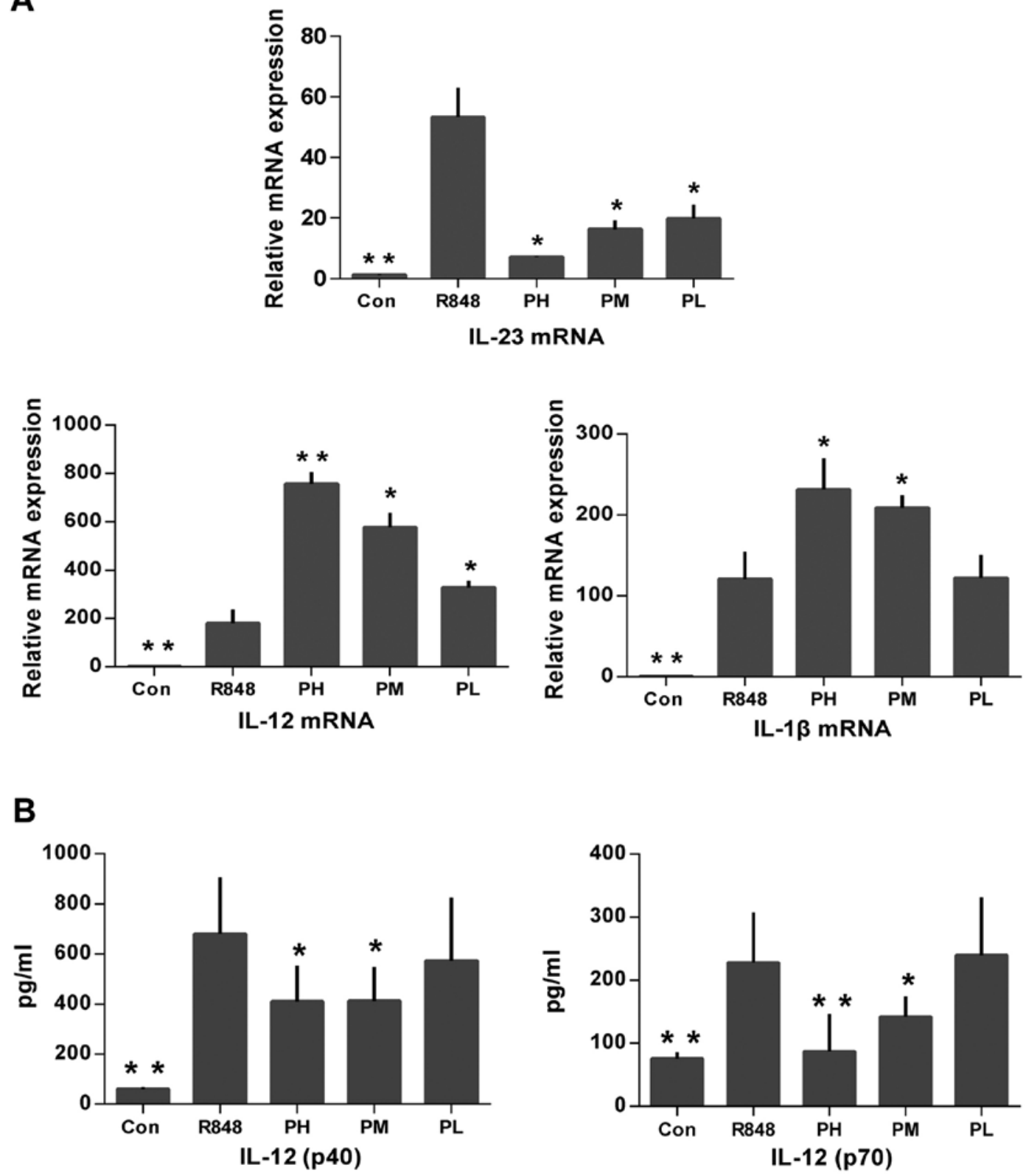

Figure 6. Paeonol decreases the expression of pro-inflammatory cytokines secreted by mature dendritic cells (DCs). (A) RT-PCR for relative expression of interleukin (IL)-23, IL-12 and IL-1 $\beta$ mRNAs in bone marrow-derived dendritic cells (BMDCs). (B) ELISA for IL-12p40 and IL-12p70 in BMDCs. Data are expressed as the mean $\pm \mathrm{SD}\left(\mathrm{n}=6 /\right.$ experiment). ${ }^{*} \mathrm{p}<0.05$ and ${ }^{* *} \mathrm{p}<0.01$ vs. the mouse model.

were decreased. IL-23, composed of two chains, the unique p19 chain and the p40 chain shared with IL-12 is required for $\mathrm{T}$ cells to produce IL-17. IL-12p40 represented IL-23 in the ELISA kit we used. Conversely, IL-12 and IL-1 $\beta$ were opposite of what we expected, which was in disagreement with a previous study (37). Strangely, groups treated with paeonol showed higher expression of IL-12 and IL-1 $\beta$ genes than the model, which was dose-dependent. Perhaps, paeonol specifically acts on the TLR7/8 signaling pathway, a MyD88dependent pathway in DCs, and inhibits the maturation and activation of DCs to reduce the secretion of IL-23. Also, we suspect that the role of paeonol varies over time.

These findings imply that paeonol may be used to treat inflammatory and hyperplastic diseases. We explored a novel strategy by which to treat psoriasis by decreasing MyD88, a key TLR adaptor protein, blocking the TLR7/8-mediated signaling pathway in DCs and contributing to the improvement of IMQ-induced psoriasis in mice.

Recent research progress on the inhibitory activity of paeonol on the inflammatory reaction is significant $(38,39)$. The mechanism by which paeonol decreases MyD88 and TLR proteins, either by inducing degradation of these proteins or blocking their synthesis, remains unknown. We will continue to explore the precise mechanisms underlying the regulation of DCs in psoriasis.

\section{Acknowledgements}

The present study was supported by the National Natural Science Foundation of China (nos. 81403410 and 81573974). 


\section{References}

1. Parisi R, Symmons DPM, Griffiths CEM and Ashcroft DM Identification and Management of Psoriasis and Associated ComorbidiTy (IMPACT) project team: Global epidemiology of psoriasis: a systematic review of incidence and prevalence. J Invest Dermatol 133: 377-385, 2013.

2. Ben Salem C, Hmouda H and Bouraoui K: Psoriasis. N Engl J Med 361: 1710, 2009.

3. Lima Ede A and Lima Mde A: Reviewing concepts in the immunopathogenesis of psoriasis. An Bras Dermatol 86 1151-1158, 2011.

4. Zaba LC, Fuentes-Duculan J, Eungdamrong NJ, Abello MV, Novitskaya I, Pierson KC, Gonzalez J, Krueger JG and Lowes MA: Psoriasis is characterized by accumulation of immunostimulatory and Th1/Th17 cell-polarizing myeloid dendritic cells. J Invest Dermatol 129: 79-88, 2009.

5. Kim J and Krueger JG: The immunopathogenesis of psoriasis. Dermatol Clin 33: 13-23, 2015.

6. Mahil SK, Capon F and Barker JN: Update on psoriasis immunopathogenesis and targeted immunotherapy. Semin Immunopathol 38: 11-27, 2016

7. Nestle FO, Turka LA and Nickoloff BJ: Characterization of dermal dendritic cells in psoriasis. Autostimulation of $\mathrm{T}$ lymphocytes and induction of Th1 type cytokines. J Clin Invest 94: 202-209, 1994

8. Nestle FO, Di Meglio P, Qin JZ and Nickoloff BJ: Skin immune sentinels in health and disease. Nat Rev Immunol 9: 679-691, 2009

9. Krueger JG and Bowcock A: Psoriasis pathophysiology: current concepts of pathogenesis. Ann Rheum Dis 64 (Suppl 2): 30-36, 2005.

10. Lowes MA, Suárez-Fariñas M and Krueger JG: Immunology of psoriasis. Annu Rev Immunol 32: 227-255, 2014

11. Di Cesare A, Di Meglio P and Nestle FO: The IL-23/Th17 axis in the immunopathogenesis of psoriasis. J Invest Dermatol 129: $1339-1350,2009$.

12. Bettelli E, Oukka M and Kuchroo VK: T(H)-17 cells in the circle of immunity and autoimmunity. Nat Immunol 8: 345-350, 2007.

13. Arican O, Aral M, Sasmaz S and Ciragil P: Serum levels of TNF-alpha, IFN-gamma, IL-6, IL-8, IL-12, IL-17, and IL-18 in patients with active psoriasis and correlation with disease severity. Mediators Inflamm 2005: 273-279, 2005.

14. Mitra A, Fallen RS and Lima HC: Cytokine-based therapy in psoriasis. Clin Rev Allergy Immunol 44: 173-182, 2013.

15. Akira S, Uematsu S and Takeuchi O: Pathogen recognition and innate immunity. Cell 124: 783-801, 2006.

16. Dika E, Varotti C, Bardazzi F and Maibach HI: Drug-induced psoriasis: an evidence-based overview and the introduction of psoriatic drug eruption probability score. Cutan Ocul Toxicol 25 : $1-11,2006$.

17. Stary G, Bangert C, Tauber M, Strohal R, Kopp T and Stingl G: Tumoricidal activity of TLR7/8-activated inflammatory dendritic cells. J Exp Med 204: 1441-1451, 2007.

18. O'Neill LA, Golenbock D and Bowie AG: The history of toll-like receptors - redefining innate immunity. Nat Rev Immunol 13: 453-460, 2013

19. Liu MH, Lin AH, Lee HF, Ko HK, Lee TS and Kou YR: Paeonol attenuates cigarette smoke-induced lung inflammation by inhibiting ROS-sensitive inflammatory signaling. Mediators Inflamm 2014: 651890, 2014

20. Chou TC: Anti-inflammatory and analgesic effects of paeonol in carrageenan-evoked thermal hyperalgesia. Br J Pharmacol 139: 1146-1152, 2003.

21. Elder JT, Bruce AT, Gudjonsson JE, Johnston A, Stuart PE, Tejasvi T, Voorhees JJ, Abecasis GR and Nair RP: Molecular dissection of psoriasis: integrating genetics and biology. J Invest Dermatol 130: 1213-1226, 2010.

22. Kim ME, Kim HK, Park HY, Kim DH, Chung HY and Lee JS: Baicalin from Scutellaria baicalensis impairs Th1 polarization through inhibition of dendritic cell maturation. J Pharmacol Sci 121: 148-156, 2013.
23. Bieber $\mathrm{K}$ and Autenrieth SE: Insights how monocytes and dendritic cells contribute and regulate immune defense against microbial pathogens. Immunobiology 220: 215-226, 2015.

24. Domingues R, de Carvalho GC, Aoki V, da Silva Duarte AJ and Sato MN: Activation of myeloid dendritic cells, effector cells and regulatory T cells in lichen planus. J Transl Med 14: 171, 2016.

25. Nahid MA, Benso LM, Shin JD, Mehmet H, Hicks A and Ramadas RA: TLR4, TLR7/8 agonist-induced miR-146a promotes macrophage tolerance to MyD88-dependent TLR agonists. J Leukoc Biol 100: 339-349, 2016.

26. Shao F, Tan T, Tan Y, Sun Y, Wu X and Xu Q: Andrographolide alleviates imiquimod-induced psoriasis in mice via inducing autophagic proteolysis of MyD88. Biochem Pharmacol 115: 94-103, 2016.

27. Yu D, Shi M, Bao J, Yu X, Li Y and Liu W: Genipin ameliorates hypertension-induced renal damage via the angiotensin II-TLR/MyD88/MAPK pathway. Fitoterapia 112: 244-253, 2016.

28. Singh M, Khong H, Dai Z, Huang XF, Wargo JA, Cooper ZA, Vasilakos JP, Hwu P and Overwijk WW: Effective innate and adaptive antimelanoma immunity through localized TLR7/8 activation. J Immunol 193: 4722-4731, 2014.

29. Singh TP, Schön MP, Wallbrecht K, Michaelis K, Rinner B, Mayer G, Schmidbauer U, Strohmaier H, Wang XJ and Wolf P: 8-Methoxypsoralen plus ultraviolet A therapy acts via inhibition of the IL-23/Th17 axis and induction of Foxp $3^{+}$regulatory T cells involving CTLA4 signaling in a psoriasis-like skin disorder. J Immunol 184: 7257-7267, 2010.

30. Zaba LC, Fuentes-Duculan J, Steinman RM, Krueger JG and Lowes MA: Normal human dermis contains distinct populations of CD11 $\mathrm{c}^{+} \mathrm{BDCA}-1^{+}$dendritic cells and $\mathrm{CD} 163^{+} \mathrm{FXIIIA}{ }^{+}$macrophages. J Clin Invest 117: 2517-2525, 2007.

31. Kollipara R, Downing C, Gordon R and Tyring S: Interleukin-23 in the pathogenesis and treatment of psoriasis. Skin Therapy Lett 20: 1-4, 2015

32. Nam KN, Woo BC, Moon SK, Park SU, Park JY, Hwang JW, Bae HS, Ko CN and Lee EH: Paeonol attenuates inflammation-mediated neurotoxicity and microglial activation. Neural Regen Res 8: 1637-1643, 2013.

33. Hsieh CL, Cheng CY, Tsai TH, Lin IH, Liu CH, Chiang SY, Lin JG, Lao CJ and Tang NY: Paeonol reduced cerebral infarction involving the superoxide anion and microglia activation in ischemia-reperfusion injured rats. J Ethnopharmacol 106: 208-215, 2006.

34. Tao W, Wang H, Su Q, Chen Y, Xue W, Xia B, Duan J and Chen G: Paeonol attenuates lipopolysaccharide-induced depressive-like behavior in mice. Psychiatry Res 238: 116-121, 2016.

35. Meng L, Xu W, Guo L, Ning W and Zeng X: Paeonol Inhibits the proliferation, invasion, and inflammatory reaction induced by TNF- $\alpha$ in vascular smooth muscle cells. Cell Biochem Biophys 73: 495-503, 2015.

36. Lin C, Lin HY, Chen JH, Tseng WP, Ko PY, Liu YS, Yeh WL and Lu DY: Effects of paeonol on anti-neuroinflammatory responses in microglial cells. Int J Mol Sci 16: 8844-8860, 2015.

37. Kim HW, Cho SI, Bae S, Kim H, Kim Y, Hwang YI, Kang JS and Lee WJ: Vitamin C up-regulates expression of CD80, CD86 and MHC class II on dendritic cell line, DC-1 via the activation of p38 MAPK. Immune Netw 12: 277-283, 2012.

38. Fu PK, Wu CL, Tsai TH and Hsieh CL: Anti-inflammatory and anticoagulative effects of paeonol on LPS-induced acute lung injury in rats. Evid Based Complement Alternat Med 2012: 837513, 2012

39. Jin X, Wang J, Xia ZM, Shang CH, Chao QL, Liu YR, Fan HY, Chen DQ, Qiu F and Zhao F: Anti-inflammatory and anti-oxidative activities of paeonol and its metabolites through blocking MAPK/ERK/p38 signaling pathway. Inflammation 39: 434-446, 2016. 